\title{
Wind Tunnel Hardware-in-the-loop Simulation Techniques for Flight Control System Evaluation
}

\author{
Min Huang, Zhongwei Wang, Zhenyun Guo, Qinghua Zeng and Yaobin Niu \\ Science and Technology on Scramjet Laboratory, National University of Defense Technology, Changsha, Hunan, 410073, China \\ ${ }^{*}$ Corresponding author
}

\begin{abstract}
In order to make up the shortages of traditional hardware-in-the-loop simulation (HILS) for flight control system (FCS) evaluation and farther reduce the risks of flight tests, wind tunnel hardware-in-the-loop simulation (WT-HILS) should be used to evaluate the FCS after the HILS evaluation and before the flight test evaluation. In this paper, a preliminary research towards the WT-HILS techniques for FCS evaluation was performed to solve several basic problems: what WT-HILS is, what FCS performances WT-HILS can evaluate, and how WTHILS will evaluate FCS. Firstly, to know what WT-HILS is, the concept, the general history and the features of WT-HILS were introduced. After that, the evaluation abilities of WT-HILS were analyzed in order to show what FCS performances WT-HILS can evaluate. Lastly, a WT-HILS evaluation method towards a classic pitching control system was designed in order to show how WTHILS will evaluate FCS.
\end{abstract}

Keywords-flight control system evaluation; hardware-in-theloop simulation; wind-tunnel hardware-in-the-loop simulation; flight test; virtual flight testing

\section{INTRODUCTION}

As the traditional hardware-in-the-loop simulation (HILS) in the laboratory for flight control system (FCS) evaluation has to build the mathematical models for aircraft aerodynamics and attitude motions, it still has a great distance away from the totally real flight tests. To replace the mathematical aerodynamic models and attitude models in HILS with real factors, wind-tunnel hardware-in-the-loop simulation (WTHILS) was used. In WT-HILS, the aerodynamic forces act on the aircraft model in real-time by putting the aircraft model under the simulated airflow in wind tunnel and the aircraft model perform the real attitude motions under the control of the real FCS as shown in FIG. I. Obviously, the WT-HILS is more realistic than the traditional laboratory based HILS. If the WT-HILS is applied to evaluate the FCS before flight tests and after HILS, some FCS errors, such as the control loss in unsteady motions that may occur in real flights and are not detected in HILS can be exposed by WT-HILS. Therefore, the risks of flight tests can be farther reduced by WT-HILS.

In recent decades, many countries were positively developing the WT-HILS techniques for FCS evaluation [1]. In USA, an eight-wire suspended 2-DOF rig developed by Arnold Engineering Development Center (AEDC) in 2002 [2] and a six wire-suspended 3-DOF rig developed by Physical Sciences Incorporation (PSI) in 2009 [3] were built to research the WTHILS systems for evaluating the FCS of air-to-air missiles.
Then, a WT-HILS platform for design and testing of visionbased control algorithms of unmanned air vehicles (UAVs) was developed by the University of Texas in 2009 [4]. In UK, a 2DOF rig which permits free roll and yaw was built by the University of Cambridge in 1999 [5] for evaluating the robust controller of the HIRM (High Incidence Research Model) [6] and an unforced pitch-axis single-degree-of-freedom rig was built by the University of Bristol in 2003 for evaluating the PID controller [7] and the dynamic state-feedback gain scheduled controller [8] for the HAWK model. In Russia, a 3DOF rig in T-203 low-speed wind tunnel was built by SibNIA in 2002 [9] for evaluating the spin recovery strategies of combat aircrafts [10]. In Sweden, a yaw-degree-of-freedom test rig was developed by the Royal Institute of Technology in 2010 [11] for evaluating the control laws of tailless aircrafts. In France, a 3-DOF (free pitch, yaw, and roll) test setup for an autonomous projectile prototype was built by the FrenchGerman Research Institute of Saint-Louis (ISL) in 2015 [12] in order to assess several control approaches for the projectile. In China, a 3-DOF (free pitch, roll, and forced yaw) rig in the $2.4 \mathrm{~m}$ transonic wind tunnel from the China Aerodynamics Research and development center (CARDC) was built in 2012 [13] for evaluating the decoupling control method of a missile [14] and the scheme of a low-speed 3-DOF rig was proposed in 2012 [15] for evaluating the control laws of various aircrafts.

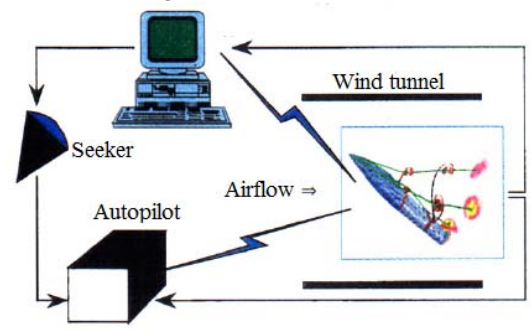

FIGURE I. SKETCH MAP OF THE WT-HILS SIMULATION

According to the above survey of the development of WTHILS, lots of rigs had been developed in an attempt to evaluate FCS. However, their researches mainly concentrated on the development of testing rigs and few analyzed the FCS evaluation abilities of WT-HILS, and how to evaluate FCS with WT-HILS. In this paper, these issues were addressed and by answering these questions, the limitation of WT-HILS for evaluating FCS can be known and a reference WT-HILS evaluation method for FCS evaluation can be provided. 


\section{EVALUTING ABILITIES OF WT-HILS}

\section{A. Features of WT-HILS}

Before researching the WT-HILS techniques, features of WT-HILS should be first pointed out. As the WT-HILS is based on wind tunnels, it has the following three obvious features:

(1) Only aerodynamic angles (angle of attack, angle of sideslip) can be reproduced in wind tunnels. Due to this feature, attitude angles (pitch angle, yaw angle, roll angle) should be calculated because attitude angles consist of not only the aerodynamic angles but also the velocity angles (flight path angle, heading angle). Therefore, the velocity angles and attitude angles should be calculated with the measured data in wind tunnels.

(2) Translational motions are limited in wind tunnels. Due to the limitation of translational motions, translational motions should be calculated with the measured data from wind tunnels.

(3) Most of current wind tunnels, especially high-speed wind tunnels cannot adjust airflow speed in real-time. Due to this feature, the FCS performances that WT-HILS can evaluate are limited.

\section{B. What FCS Performances WT-HILS Can Evaluate?}

Generally, FCS performances can be described as the flight performances and flight qualities under the action of FCS. The flight performances are mainly used for describing the characteristics of translational motions, including velocity indexes, height indexes, taking off and landing indexes, maneuvering indexes, endurance indexes, guidance precision, flight envelope and so on [16-17]. The flight qualities are mainly used for describing the characteristics of rotational motions, including longitudinal flight qualities, lateraldirectional flight qualities, flight qualities under unsteady maneuvers, agility indexes and so on [17-18].

As the airflow speed cannot be changed in WT-HILS, the FCS performances that WT-HILS can evaluate are analyzed as follows.

(1) Flight performances in steady flight. As in steady flight the translational accelerations are 0 , flight performances under such flight condition can be evaluated by WT-HILS, such as the target tracking indexes in steady flight, the hovering radius and cycle indexes in steady hover. While due to the limitation of speed change, the velocity indexes, taking off and landing indexes, guidance precision, and flight envelope cannot be evaluated.

(2) Longitudinal short-term performances, stability and control performances of lateral-directional motions. Under normal motion conditions (motions with small amplitude), the aircraft motions can be decoupled into longitudinal motions and lateral-directional motions. And the longitudinal motions include the short-term mode and long-term mode. The shortterm mode is relative to angle of attack and pitch angle and the influence of velocity can be neglected. The long-term mode is mainly relative to the velocity and flight path angle. Lateraldirectional motions include the Dutch roll mode, roll mode and spiral mode. These modes are mainly relative to roll angle, angle of sideslip and yaw angle and the influence of velocity can be neglected. Therefore, longitudinal short-term performances, stability and control performances of lateraldirectional motions can be evaluated by WT-HILS, while longitudinal long-term performances cannot be evaluated.

(3) FCS performances of unsteady maneuvers. The unsteady maneuvers, which are mainly relative to large angle of attack or large angle of sideslip, have quite obvious nonlinear aerodynamic characteristics and have strong couplings between aerodynamics and motions. To modeling these maneuvers realistically, the WT-HILS rig should permit free rotations or free maneuvers so as to avoid the calculating of aerodynamic angles with measured moments and the aerodynamics and motions can counteract with each other as that of real flights. In this way, FCS performances of unsteady maneuvers can be effectively evaluated by WT-HILS. Some departments had developed rigs to evaluate the control algorithms of free spin [9-10], free wing rock [11], free pitching-rolling coupling motions [14].

(4) Heading agility indexes. Aircraft agility can be divided into flight path agility and heading agility. The flight path agility means "the ability to change the direction and amplitude of velocity with FCS”. Apparently, it cannot be evaluated by WT-HILS. The heading agility means "the ability to change the head directions (such as pitch and yaw) with FCS”. It is mainly relative to the attitudes and maneuvering time and can be evaluated by WT-HILS.

\section{An EVAluation Method OF WT-HILS}

Concluded from the evaluation processes of numerical simulations, HILS and flight tests, the technical content of a WT-HILS evaluation method can be divided into four steps (shown in FIG. II): 1) definite the FCS performances and indexes needed to be evaluated; 2) aiming at the FCS indexes, design the WT-HILS test method, which should definite the parameters need to be measured, the test system scheme, test types and test procedures; 3) design the data processing method, which should transfer the measured data to the indexes; 4) design the performance determination method, which should judge whether the indexes are satisfied or have a enough performance level. The following is a WT-HILS evaluation method towards a pitching control system [19].

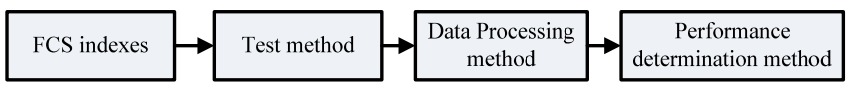

FIGURE II. PROCEDURES OF A WT-HILS EVALUATION METHOD

\section{A. Evaluation Object: a Pitching Control System}

The pitching control system of an unmanned aircraft is taken as the evaluation object (shown in FIG. III). By inputting the desired pitch angle $\varphi_{i}$ and the feedback angle $\varphi_{o}$ to the pitch controller, a control signal, namely an elevator deflection angle $\delta$ is generated. Then the aircraft will response to the deflection of pitching elevators and perform the pitch motion. The controller will keep adjusting the $\delta$ until the $\varphi_{o}$ reaches to the $\varphi_{i}$. 


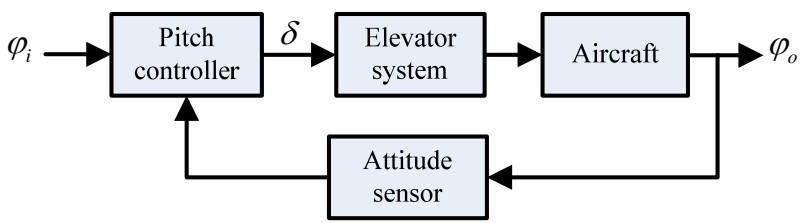

FIGURE III. PITCHING CONTROL SYSTEM OF AN UNMANNED AIRCRAFT

The WT-HILS can evaluate whether the pitching control system can accurately tracks the pitch command $\varphi_{i}$. Three indexes are used to represent the attitude tracking performance of this control system: $e_{t r}, t_{s}, \sigma$.

\section{B. WT-HILS Evaluation Method for Evaluating the Pitching Control System}

\section{1) Test method}

The test system scheme for evaluating the pitching control system is shown in FIG. IV. The FCS hardware, including the controller, the sensor and the servo, are placed inside the aircraft test article as in flight tests. The test article is fixed on a sting support which drives the test article to perform angle of attack motions. The sensors measure angle of attack, lift $L$, pitching moment $M_{z}$. With the measured pitching moment, the RCAS calculates the angle of attack command $\alpha_{i}$ for the sting support. With the measured angle of attack and lift, the RCAS calculates the feedback pitch angle $\varphi_{o}$ for the controller.

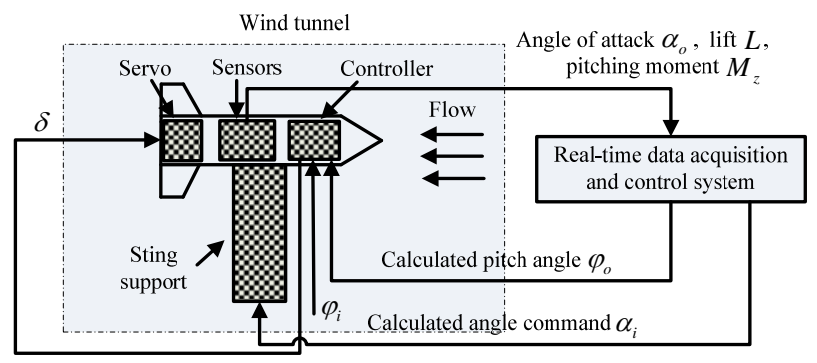

FIGURE IV. A WT-HILS TEST SYSTEM SCHEME FOR EVALUATING THE PITCHING CONTROL SYSTEM

The equation for calculating $\alpha_{i}$ [20] is:

$$
\ddot{\alpha}_{i}=M_{z} / I_{z}
$$

The equation for calculating $\varphi_{o}[21]$ is:

$$
\left\{\begin{array}{c}
\dot{\theta}=\frac{T \sin \alpha_{i}+L}{m V}-\frac{g \cos \theta}{V} \\
\varphi_{0}=\theta+\alpha_{i}
\end{array}\right.
$$

With the test system above, the closed-loop attitude control test should be performed to obtain the attitude tracking indexes. The input command is the classic step signal shown in FIG. V.

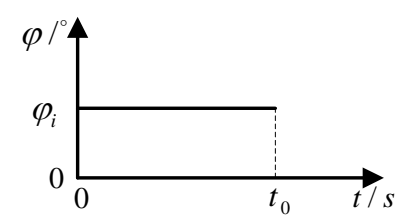

FIGURE V. THE INPUT COMMAND

The test procedure is as follows:

a) Preload the input command into the controller;

b) Check whether the system works well, if the system works well, turn on the system;

c) When the wind tunnel airflow is steady, the RACS begins to work and continuously collects data, processes data, sends the calculated $\varphi_{o}$ to the controller, and controls the support rig to the new angle of attack $\alpha_{i}$;

d) Watch the motion of the aircraft test article, when the test article almost stays at an angle or even gets diverged, lock the support rig and close the wind tunnel.

\section{2) Data processing method}

After finishing the above test, the RCAS records a series of time-varying pitch angles which can be integrated as an attitude response curve. From the curve, the $\varphi_{m}, \varphi_{e}$, and $t_{s}$ can be read. Then, the $\sigma$ and $e_{t r}$ can be calculated with the following equations:

$$
\begin{aligned}
& \sigma=\left|\varphi_{m}-\varphi_{e}\right| / \varphi_{e} \times 100 \% \\
& e_{t r}=\left|\varphi_{e}-\varphi_{c}\right| / \varphi_{c} \times 100 \%
\end{aligned}
$$

\section{3) FCS performance determination method}

After obtaining the FCS indexes, the index level should be determined. Here, two steps are used to determine the index level: the general region requirement and the level dividing. First, it should be judged whether the index is in the general region. If the index is in the general region, the level of the index should be divided then. Here, three levels are used. Their meanings are listed in TABLE 1 . To different vehicles, the required region of each index is different. For the sake of later simulation demonstration, a sample general region and each level region are listed in TABLE 2. These regions were assumed and were not to a specific aircraft, but were chosen by resembling realistic metrics. 
TABLE I. MEANINGS OF EACH LEVEL

\begin{tabular}{ll}
\hline Level & Content \\
\hline 1 & $\begin{array}{l}\text { Satisfying: the index is obviously suitable for performing } \\
\text { flight missions }\end{array}$ \\
\hline 2 & $\begin{array}{l}\text { Acceptable: the index is suitable for performing flight } \\
\text { missions, but the efficiency is decreased }\end{array}$ \\
\hline 3 & $\begin{array}{l}\text { Controllable: the index is suitable for safe flight, but cannot } \\
\text { ensure finishing missions }\end{array}$ \\
\hline
\end{tabular}

TABLE II. A SAMPLE GENERAL REGION AND EACH LEVEL REGION OF FCS INDEXES

\begin{tabular}{lllll}
\hline \multirow{2}{*}{$\begin{array}{l}\text { Performance } \\
\text { indexes }\end{array}$} & General & \multicolumn{2}{l}{ Level dividing } & \\
\hline$e_{t r}$ & {$[0,3 \%]$} & {$[0,1 \%]$} & $(1 \%, 2 \%]$ & $(2 \%, 3 \%]$ \\
\hline$t_{s}$ & $(0,1]$ & $(0,0.3]$ & $(0.3,0.6]$ & $(0.6,1]$ \\
\hline$\sigma$ & {$[0,145 \%]$} & {$[0,45 \%]$} & $(45 \%, 100 \%]$ & $(100 \%$, \\
\hline
\end{tabular}

C. Numerical Simulation Demonstration of the WT-HILS evaluation method

\section{1) Numerical simulation scheme}

To illustrate the WT-HILS evaluation method quantitatively, a numerical simulation of WT-HILS was performed with a sample air vehicle. The numerical simulation scheme is shown in FIG. 6, in which the elevator actuator model and the sensor model are not considered.

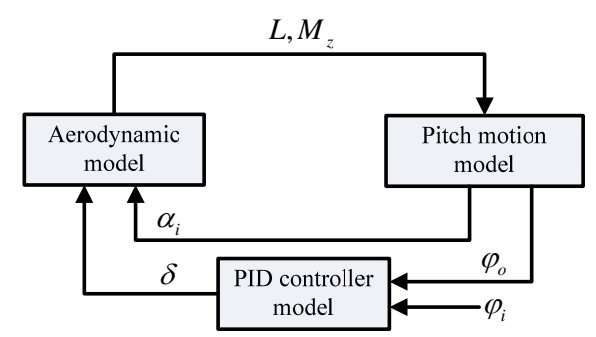

FIGURE VI. NUMERICAL SIMULATION SCHEME OF THE WT-HILS SYSTEM

The pitch motion model is a combination of the equations (1)-(2).

Thereinto, the $L$ and $M_{z}$ can be calculated with the following aerodynamic model:

$$
\left\{\begin{array}{c}
L=q S m_{l} \\
M_{z s}=m_{z s} q S l \alpha_{i} \\
M_{z d}=m_{z d} q S l\left(0.5 \dot{\alpha}_{i} l / V\right) \\
M_{z}=M_{z s}+M_{z d}
\end{array}\right.
$$

The $m_{l}, m_{z s}$ and $m_{z d}$ corresponding to any state of $\alpha_{i}$ and $\delta$ can be obtained by aerodynamic tables.

The PID controller model [22] is:

$$
\delta(t)=K_{p} e(t)+K_{i} \int_{0}^{t} e(\tau) d \tau+K_{d} \frac{d e(t)}{d t}
$$

\section{2) Simulation and performance determination}

The attitude tracking performance was evaluated in the following steps.

a. Numerical simulation of WT-HILS and data collection. Set the initial angle of attack, rate of angle of attack, and elevator deflection as 0 . Set the input $\varphi_{i}=1^{\circ}$, and start the simulation of WT-HILS closed-loop attitude control test. The $\varphi_{o}$ was collected shown in FIG. 7.

b. Data processing. From the curve of $\varphi, \varphi_{m}, \varphi_{e}$, and $t_{s}$ were read. Then, $\sigma$ and $e_{t r}$ were calculated: $\sigma=14.6 \%$, $e_{t r}=0.8 \%$.

c. Performance determination. According to TABLE 2, the levels of attitude tracking indexes were determined shown in TABLE 3.

d. Analysis of the evaluation result. From TABLE 3, it is known that the controller can guarantee the FCS follow the input command effectively, but still has improving space. By adjusting the controller parameters to reduce $t_{s}$, the attitude tracking performances will be better.

From the above numerical demonstration of the designed WT-HILS evaluation method, the attitude tracking performance is successfully evaluated, which validates the feasibility of the WT-HILS evaluation method.

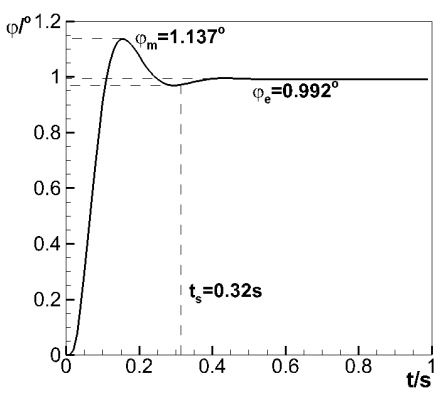

FIGURE VII. COLLECTED $\varphi_{o}$

TABLE III. THE EVALUATION RESULT OF ATTITUDE TRACKING PERFORMANCE

\begin{tabular}{llll}
\hline Indexes & $\sigma$ & $t_{s}$ & $e_{t r}$ \\
\hline Level & 1 & 2 & 1 \\
\hline
\end{tabular}

IV. CONCLUSIONS

Several basic issues concerning the WT-HILS technique for evaluating the hardware integrated FCS before flight tests and after HILS were addressed in this paper. They are summarized as follows. 
(1) According to the features of WT-HILS, the WT-HILS evaluation abilities are limited: the steady flight performances, longitudinal short-term performances, stability and control performances of lateral-directional motions, FCS performances of unsteady maneuvers, and heading agility indexes can be evaluated by WT-HILS, while the FCS performances relating to speed change, such as the guidance precision, longitudinal long-term performances and flight path agility cannot be evaluated.

(2) Aiming at the attitude tracking indexes of a classic pitching control system, a WT-HILS evaluation method was proposed, including the test method, the data processing method, and the FCS performance determination method. Then, a numerical simulation of the WT-HILS test was performed to illustrate the WT-HILS evaluation method. The simulation preliminarily validated the viability of this WTHILS evaluation method. Future work will be focusing on the wind tunnel tests to further validate the WT-HILS evaluation method.

\section{NOMENCLATURE}

$\varphi, \varphi_{i}, \varphi_{0}$ : Pitch angle, Pitch angle input, pitch angle output

$\alpha, \alpha_{i}, \dot{\alpha}_{i}, \ddot{\alpha}_{i}, \alpha_{o}$ : Angle of attack, Calculated angle of attack, rate of angle of attack, acceleration of angle of attack, measured angle of attack

\section{$\theta, \dot{\theta}$ : Flight path angle, rate of flight path angle}

\section{$\delta$ : Rudder deflection angle}

$\varphi_{m}, \varphi_{e}$ : Maximum value of the output of pitch angle, steady-state value of the output of pitch angle

$e, e_{t r}$ : Tracking error, steady-state error which is defined as the difference between $\varphi_{c}$ and $\varphi_{e}$

$t_{s}$ : Transient time, defined as the time required for the output of pitch angle reaching to $98 \%$ or $102 \%$ of the $\varphi_{e}$

$\sigma$ : Overshoot, defined as the difference between $\varphi_{m}$ and $\varphi_{C}$

$M_{z}, M_{z s}, M_{z d}, m_{z s}, m_{z d}$ : Pitching moment, steady moment in pitch, damping moment in pitch, static derivative in pitch, dynamic derivative in pitch

$L, m_{l}, T$ : Lift force, coefficient of lift force, thrust

$I_{z}, m, g$ : Inertial moment in pitch, mass of aircraft, acceleration of gravity

\section{$V$ : flight velocity}

$q, S, l$ : Dynamic pressure, maximum cross section area of the aircraft body, characterictic length of the aircraft body

$K_{p}, K_{i}, K_{d}$ : Proportional parameter, integral parameter, and differential parameter of the PID controller

\section{REFERENCES}

[1] Huang M, Wang Z W. A review of wind tunnel based virtual flight testing techniques for evaluation of flight control systems. International Journal of Aerospace Engineering, 2015.

[2] Lawrence C, Mills B. Status update of the AEDC virtual flight testing development program. AIAA 2002-0168, 2002.

[3] Magill J C, Cataldi P, Morency J R, Hammer D X, Burgess R, Jeter E. Demonstration of a wire suspension for wind-tunnel virtual flight testing. Journal of Spacecraft and Rockets, 2009, 46(3): 624-633.

[4] Gans N R, Dixon W E, Lind R, Kurdila A. A hardware in the loop simulation platform for vision-based control of unmanned air vehicles. Mechatronics, 2009, 19(2009): 1043-1056.

[5] Papageorgiou G, Glover K. Design, development and control of the HIRM wind tunnel model. Proceedings of the 38th IEEE conference on decision and control, 1999.

[6] Papageorgiou G, Glover K. Two-degree-of-freedom control of an actively controlled wind-tunnel model. Journal of Guidance Control and Dynamics, 2002, 25(3): 510-516.

[7] Davison P M, Bernardo M D, Lowenberg M H. Modeling and control of a single degree of freedom dynamic wind tunnel rig. Proceedings of the European control conference, 2003.

[8] Richardson $\mathrm{T} \mathrm{S}$, Dubs A, Lowenberg $\mathrm{M} \mathrm{H}$, Jones C D. Wind-tunne testing of a dynamic state-feedback gain scheduled control system. AIAA 2005-5976, 2005.

[9] Sohi N P, Prudnikov Y A, Temlyakov Y N. Estimation of spin characteristics of aerobatic aircraft by means of spin modes modeling in a horizontal wind tunnel. 22th international congress of the aeronautical sciences, 2002.

[10] Sohi N P. Modeling of spin modes of supersonic aircraft in horizonta wind tunnel. 24th International Congress of the Aeronautical Sciences, 2004.

[11] Stenfelt G, Ringertz U. Yaw control of a tailless aircraft configuration. Journal of aircraft, 2010, 47(5): 1807-1810.

[12] Strub G, Theodoulis S, Gassmann V, Dobre S. Pitch Axis Control for a Guided Projectile in a Wind Tunnel-based Hardware-In-the-Loop Setup. AIAA Modeling and Simulation Technologies Conference, AIAA 20150153, 2015.

[13] Li H. Study on the similarity critiria and simulation method of the wind tunnel based virtual flight testing. PhD Thesis, China Aerodynamics Research and Development Center, 2012. (in Chinese)

[14] Zhao Z L, Wu J Q, L H, Zhou W Q, Mao D Y, Yang H Y. Investigation of virtual flight testing technique based on $2.4 \mathrm{~m}$ transonic wind tunnel. Acta Aeronautica et Astronautica Sinica, 2016, 37(2): 504-512. (in Chinese)

[15] Nie B W, Zhu M H, Guo L L, Wen Y C, Jiang M. Key technique and design scheme of the wind tunnel virtual flight system. 2012 Chinese guidance, navigation and control conference (CGNCC2012), Beijing, 2012. (in Chinese)

[16] Chen T N. Aircraft flight performance quality and control. Beijing: National Defense Industry Press, 2007. (in Chinese)

[17] Zeng Q H, Guo Z Y. Unmanned flight control techniques and engineering. Beijing: National Defense Industry Press, 2011. (in Chinese)

[18] Gao J Y, Li L Y, Feng Y C, etc. Aircraft flight qualities. Beijing: National Defense Industry Press, 2011. (in Chinese)

[19] Huang M, Wang Z W, Guo Z Y, Niu Y B. Design of the wind tunne based virtual flight testing evaluation method for flight control systems. Proc IMechE Part G: Journal of Aerospace Engineering, 2016, 0(0): 113.

[20] Li H, Zhao Z L, Fan Z L. Simulation method for wind tunnel based virtual flight testing. International Journal of Modern Physics: Conference Series, 2012, 19(2012): 381-389.

[21] Li H F. Guidance and control techniques of hypersonic aircrafts. Beijing Chinese aerospace press, 2012. (in Chinese)

[22] Huang Y R, Qu L G. Parameter adjusting and realizing of PID controllers. Beijing: Science press, 2010. (in Chinese) 